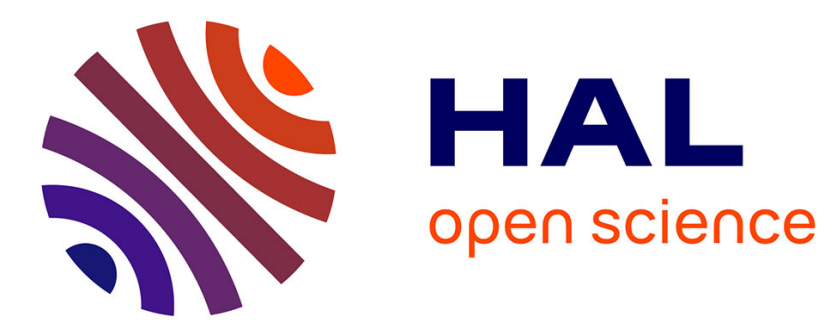

\title{
Guide on set invariance for delay difference equations
}

Mohammed-Tahar Laraba, Sorin Olaru, Silviu-Iulian Niculescu, Franco

Blanchini, Giulia Giordano, Daniele Casagrande, Stefano Miani

\section{To cite this version:}

Mohammed-Tahar Laraba, Sorin Olaru, Silviu-Iulian Niculescu, Franco Blanchini, Giulia Giordano, et al.. Guide on set invariance for delay difference equations. Annual Reviews in Control, 2016, 41, pp.13 - 23. 10.1016/j.arcontrol.2016.04.020 . hal-01408474

\section{HAL Id: hal-01408474 \\ https://hal-centralesupelec.archives-ouvertes.fr/hal-01408474}

Submitted on 5 Dec 2016

HAL is a multi-disciplinary open access archive for the deposit and dissemination of scientific research documents, whether they are published or not. The documents may come from teaching and research institutions in France or abroad, or from public or private research centers.
L'archive ouverte pluridisciplinaire HAL, est destinée au dépôt et à la diffusion de documents scientifiques de niveau recherche, publiés ou non, émanant des établissements d'enseignement et de recherche français ou étrangers, des laboratoires publics ou privés. 


\title{
Guide on Set Invariance for Delay Difference Equations ${ }^{\text {th }}$
}

\author{
Mohammed-Tahar Laraba ${ }^{\mathrm{a}}$, Sorin Olaru ${ }^{\mathrm{a}}$, Silviu-Iulian Niculescu ${ }^{\mathrm{a}}$, Franco Blanchini ${ }^{\mathrm{b}}$, Giulia Giordano $^{\mathrm{b}}$, \\ Daniele Casagrande ${ }^{c}$, Stefano Mianic \\ ${ }^{a}$ Laboratory of Signals and Systems(L2S, UMR CNRS 8506), CentraleSupeléc-CNRS-Université Paris-Saclay, France \\ ${ }^{b}$ Department of Mathematics and Computer Science, Univ. of Udine, Italy. \\ ${ }^{c}$ Department of Electrical, Management and Mechanical Engineering, Univ. of Udine, Italy.
}

\begin{abstract}
This paper addresses set invariance properties for linear time-delay systems. More precisely, the first goal of the article is to review known necessary and/or sufficient conditions for the existence of invariant sets with respect to dynamical systems described by linear discrete time-delay difference equations (dDDEs).

Secondly, we address the construction of invariant sets in the original state space (also called $\mathcal{D}$-invariant sets) by exploiting the forward mappings. The notion of $\mathcal{D}$-invariance is appealing since it provides a region of attraction, which is difficult to obtain for delay systems without taking into account the delayed states in some appropriate extended state space model.

The present paper contains a sufficient condition for the existence of ellipsoidal $\mathcal{D}$-contractive sets for dDDEs, and a necessary and sufficient condition for the existence of $\mathcal{D}$-invariant sets in relation to linear time-varying dDDE stability. Another contribution is the clarification of the relationship between convexity (convex hull operation) and $\mathcal{D}$-invariance of linear dDDEs. In short, it is shown that the convex hull of the union of two or more $\mathcal{D}$-invariant sets is not necessarily $\mathcal{D}$-invariant, while the convex hull of a non-convex $\mathcal{D}$-invariant set is $\mathcal{D}$-invariant.
\end{abstract}

Keywords: Set invariance, Linear time-delay systems, Discrete time-delay difference equations.

\section{Introductory remarks}

Positive invariance is an essential concept in control theory, with applications to constrained dynamical systems analysis, uncertainty handling as well as related control design problems $[1,2,3]$. It serves as a basic tool in many topics, such as model predictive control [4, 5, 6], fault tolerant control [7] and reference governor design [8]. Furthermore, there exists a close link between classical stability theory and positive invariant sets. It is worth mentioning that, in Lyapunov theory, invariance is implicitly described

${ }^{\sqrt{3}}$ A preliminary version of the paper has been presented at the $12^{\text {th }}$ IFAC Workshop on Time Delay Systems, Ann Arbor (MI), USA, 2015.

Email addresses: mohammed.laraba@lss.supelec.fr

(Mohammed-Tahar Laraba), sorin.olaru@lss.supelec.fr

(Sorin Olaru), silviu.niculescu@lss.supelec.fr

(Silviu-Iulian Niculescu)

$U R L$ :

http: //www.supelec.fr/360_p_40423/mohammed-tahar-laraba

(Mohammed-Tahar Laraba) by the sub-level sets of a Lyapunov function, which are known to be contractive sets [9].

The response of a dynamical system to external excitation is rarely instantaneous, and time-delay models are well suited for describing dynamics related to propagation phenomena and/or communication flows (see, for example, $[10,11,12,13,14,15,16,17,18$, $19,20,21,22,23])$. In closed loop, the dynamics can be represented by delay differential equations (resp. inclusions) or delay difference equations (resp. inclusions) according to the continuous/discrete framework and the presence of disturbances or uncertainties. In the present paper, we consider autonomous dynamics where the delayed arguments are treated as a state dependence and not as a perturbation signal.

From a mathematical point of view, delay difference equations form an important modeling class, since most modern controllers are implemented via computers or dedicated embedded systems. They have been widely studied in the literature (see [24, $25,26,27])$. Difference and differential equations 
with unbounded random delays have been addressed in [28]. Delay difference inclusions DDIs represent also a rich modeling class including networked control systems and uncertain time-delay systems. The relationship between stability of DDIs and the existence of Lyapunov-Krasovskii and Lyapunov-Razumikhin functions has been studied in detail in [29]. Stabilizing controller construction and stability analysis based on Lyapunov-Krasovskii and LyapunovRazumikhin functions for DDIs have been proposed therein.

Positive invariance for dynamical systems described by dDDEs has been addressed in [30]. As hinted before, two main approaches exist in the literature dealing with positive invariant sets for discrete time-delay difference equations. The first approach, referred to as Krasovskii approach, relies on the fact that the discrete-time dDDE allows a finitedimensional extended state space model (this representing a demarcation with respect to the continuoustime counterpart). This extended state space, whose dimension is finite but strongly related to the delay value, leads to an invariant set characterization with respect to an equivalent linear time-invariant model. This concept is well understood and popular in the literature, but it suffers from an increased numerical complexity when delays are relatively large. Lyapunov-Krasovskii and spectral techniques have been also used in [31] to analyze Lyapunov and asymptotic stability.

The second approach, referred to as Razumikhin approach, has been formulated in the '90s and reinvestigated in the last decade, to obtain an invariant set for the dDDE in the original state space, which is independent of the delay value. This concept is also denoted as $\mathcal{D}$-invariance, and is often conservative as long as the existence conditions are restrictive. It is worth mentioning that a relaxation of the Lyapunov-Razumikhin conditions has been proposed by [32]. The proposed conditions, which can be verified by solving an LMI problem for linear dDDEs, prove to be necessary and sufficient for asymptotic stability of dDDEs. Furthermore, the obtained relaxed Lyapunov-Razumikhin functions are useful for constructing invariant sets for dDDEs.

It has been recently recognized that $\mathcal{D}$-invariance can be seen as set factorization of an invariant set in the extended state space [33]. It has been established that the extended state space invariance corresponds to a minimal factorization while $\mathcal{D}$-invariance, under the constraints imposed by the dimension of the dDDE, represents the maximal regular ordered factorization. This interesting result opens the way for factorizations which are in between the two representations, by exploiting non-minimal state space equations. In [34], the authors have focused on the maximal factorizations. They have proposed a characterization of the link between the Razumikhin and Krasovskii approaches, by using set factorization. The proposed framework yields a fitting trade-off between the conceptual generality of the extended state space approach and the computational convenience of the $\mathcal{D}$-invariance approach. It has been shown that $\mathcal{D}$-invariance represents a particular realization of a broader family of invariant structures. The relationship between these families of invariant sets has been established via set factorization and conjugacy. In [35], two specific families of controlled $(\mathrm{k}, \lambda)$ contractive sets in the augmented state space framework have been characterized and the link between these controlled $(k, \lambda)$-contractive sets and those of the time-delay system has been established in [36].

In [37], a new concept of set invariance with respect to discrete-time linear systems subject to delays has been introduced. A family of sets which represent a sequence of cyclically invariant subsets of the state space was defined and characterized. Basically, the existing algebraic conditions for invariance analysis of linear dynamics have been generalized and conditions for the invariance of a given sequences of sets with respect to linear discrete-time dynamics in the presence of delay have been established. The notion of invariant family of sets has been proposed in $[38,36]$ to generalize the cyclic invariance concept.

This paper is an extended version of work published in [39], where we addressed the existence of positive invariant sets in the state space of the original dDDE. More precisely, the case of two delays was addressed in the conference paper, while the general case is treated here. $\mathcal{D}$-invariant sets can be seen as invariant sets in both the current and the retarded state space and further related to the stability analysis based on Lyapunov-Razumikhin approach. Sufficient conditions for the existence of a $\mathcal{D}$-invariant set have been first obtained in $[40,41]$. Then, a necessary and sufficient characterization for the existence of $\mathcal{D}$ invariant sets has been provided in $[42,43]$. Particularly, as far as the construction of $\mathcal{D}$-invariant sets is concerned, we can find a series of results in [44, 45], which will be appropriately recalled in the present 
paper. Recently, [46] has proposed a computationally efficient numerical routine which is necessary to guarantee the existence of $\mathcal{D}$-invariant sets for the delay difference equations with two delay parameters. This condition covers, for the two delay case, the existing necessary conditions in the literature and proves to reduce considerably the gap with respect to sufficient conditions. In the present work, we provide an interesting example for which the condition in [46] is verified but the existing algorithms fail to construct a $\mathcal{D}$-invariant set.

As discussed in [47], from the stability point of view a pertinent analysis of $\mathcal{D}$-invariance can be made in relationship with delay-independent stability. In short, it has been shown that the existence of a diagonal Lyapunov-Krasovskii functional is necessary and sufficient for delay-independent stability. Polyhedral Lyapunov functions have been used for stability and positive invariance analysis of networked control systems in the presence of bounded delays, constant, unknown or time-varying. The problem of finding stability margins has been proved to reduce to a linear programming problem [48].

To summarize, the main objectives of the present paper are resumed as follows: i) an overview of necessary and/or sufficient conditions for the existence of $\mathcal{D}$-invariant sets for dDDEs with an arbitrary delay value; ii) a sufficient condition for the existence of ellipsoidal $\mathcal{D}$-invariant sets for dDDEs; iii) the proof of the relationship between time-varying dDDE stability and the existence of $\mathcal{D}$-invariant sets; iv) the proof of two properties related to convexity and convex operations over $\mathcal{D}$-invariant sets. Notably, it is established that a dDDE admits a $\mathcal{D}$-invariant set if and only if it is time-varying delay-independent stable.

This paper is structured as follows. Section 2 presents some preliminary mathematical notions and definitions. Basic properties of $\mathcal{D}$-invariance concept are addressed in Section 3. In the same section, we present necessary and sufficient conditions for the existence of non trivial sets. The relationship between $\mathcal{D}$-invariance and stability of dDDEs concludes the section. Algorithmic construction based on set iteration using forward mappings, and some illustrative examples are revisited in Section 4. The concepts of cyclic invariance and the invariant families of sets as well as the relationship with the set factorization are presented in Section 5. Finally Section 6 draws some concluding remarks.

\section{Prerequisites}

\subsection{Notations}

We denote by $\mathbb{R}, \mathbb{R}_{+}, \mathbb{Z}$ and $\mathbb{Z}_{+}$sets of real numbers, non-negative reals, integer numbers and nonnegative integers, respectively. For every interval $\Pi$ of $\mathbb{R}$ we define $\mathbb{Z}_{\Pi}:=\mathbb{Z} \cap \Pi$. For an arbitrary set $\mathcal{A} \subseteq \mathbb{R}^{n}$, int $(\mathcal{A})$ denotes the interior of $\mathcal{A}$. $\mathbb{B}_{r}^{n}(0)$ denotes the ball of radius $r$ in Euclidean norm, centered in the origin of $\mathbb{R}^{n}$. We denote by $\mathbf{1}_{n}$ the vector of dimension 'n' with all the entries equal to 1 . We denote by $\mathbb{D}, \partial \mathbb{D}, \operatorname{ext}(\mathbb{D})$ the open unit disc, the unit circle and the exterior of the closed unit disc respectively. For the matrix pair $(A, B)$, the set of generalized eigenvalues and the Kronecker product are denoted by $\gamma(A, B)$ and $A \otimes B$, respectively. $I_{n} \in \mathbb{R}^{n \times n}$ and $0_{n \times m} \in \mathbb{R}^{n \times m}$ denote the identity and the null matrix, respectively. $\mathcal{X} \oplus \mathcal{Y}$ denotes the Minkowski sum of sets $\mathcal{X}$ and $\mathcal{Y}$, it is defined by:

$\mathcal{X} \oplus \mathcal{Y}:=\{z \mid \exists(x, y) \in(\mathcal{X}, \mathcal{Y})$ such that $z=x+y\}$.

Definition 1. A set $\mathcal{P} \subseteq \mathbb{R}^{n}$ is bounded if there exists $r \in \mathbb{R}_{+}$such that $\overline{\mathcal{P}} \subset \mathbb{B}_{r}^{n}(0)$; closed if $\forall x \notin$ $\mathcal{P}, \exists \epsilon \in \mathbb{R}_{+}$such that $\mathbb{B}_{\epsilon}^{n}(x) \cap \mathcal{P}=\emptyset$; compact if it is bounded and closed.

Definition 2. A set $\mathcal{P} \subseteq \mathbb{R}^{n}$ is a (proper) $\mathcal{C}$-set if is convex, compact and includes the origin in its strict interior.

We denote by $\operatorname{Com}\left(\mathbb{R}^{n}\right)$ and $\operatorname{Com} C\left(\mathbb{R}^{n}\right)$ the space of compact subsets and the space of $\mathcal{C}$-subsets of $\mathbb{R}^{n}$ containing the origin, respectively. The spectrum of a matrix $A \in \mathbb{R}^{n \times n}$ is the set of the eigenvalues of $A$, denoted by $\lambda(A)$, while the spectral radius is defined as $\rho(A):=\max _{\xi \in \lambda(A)}(|\xi|)$. The spectral norm will be denoted by $\sigma(A)$ and is defined as $\sigma(A):=\sqrt{\rho\left(A^{T} A\right)}$.

\subsection{System Dynamics}

In the sequel, we will consider discrete time-delay difference equations of the form:

$$
x(k+1)=\sum_{i=0}^{d} A_{i} x(k-i)
$$

where $x(k) \in \mathbb{R}^{n}$ is the state vector at the time $k \in$ $\mathbb{Z}_{+}, d \in \mathbb{Z}_{+}$is the maximal fixed time-delay, the matrices $A_{i} \in \mathbb{R}^{n \times n}$, for $i \in \mathbb{Z}_{[0, d]}$ and the initial conditions are considered to be given by $x(-i)=x_{-i} \in \mathbb{R}^{n}$, for $i \in \mathbb{Z}_{[0, d]}$. 
Definition 3. The null solution of the dDDE:

$$
x(k+1)=\sum_{i=0}^{m} A_{i} x\left(k-d_{i}\right)
$$

is asymptotically stable if $\forall \epsilon>0, \exists \delta>0$ such that whenever $\sup _{j}\|x(-j)\| \leq \delta, j=\{1, \cdots, m\}$, $\|x(k)\|<\epsilon, \forall k \in \mathbb{Z}_{+}$and $x(k) \rightarrow 0$ when $k \rightarrow \infty$.

Definition 4. The dDDE (2) is delay-independently stable if its null solution is stable $\forall d=\left[d_{0} \cdots d_{m}\right] \in$ $\left(\mathbb{Z}_{+}\right)^{m+1}$.

Definition 5. The dDDE with time-varying (positive) delay values:

$$
x(k+1)=\sum_{i=0}^{m} A_{i} x\left(k-d_{i}(k)\right)
$$

is delay-independently stable if its null solution is stable $\forall d(k)=\left[d_{0}(k) \cdots d_{m}(k)\right] \in\left(\mathbb{Z}_{+}\right)^{m+1}$.

It is clear that an extended state space representation can be constructed for any given delay realization. For instance, by setting $\xi(k)=$ $\left[x(k)^{T} \cdots x(k-d)^{T}\right]^{T}$, equation (1) can be rewritten as:

$$
\xi(k+1)=A_{\xi} \xi(k)=\left[\begin{array}{cccc}
A_{0} & \ldots & A_{d-1} & A_{d} \\
I & \ldots & 0 & 0 \\
\vdots & \ddots & \vdots & \vdots \\
0 & \ldots & I & 0
\end{array}\right] \xi(k)
$$

This class is relevant for modeling several propagation and transmission phenomena. One example is represented by networked control systems (see [49]) where the feedback mechanism is affected by communication delays. These delays are known to degrade the performances and eventually affect stability [50]. We report next, without proof, some well-known results related to asymptotic stability of systems (1) and (4) (see e.g [51]).

Lemma 1. The following statements hold:

- System (1) is asymptotically stable if and only if

$$
\operatorname{det}\left(z I-\sum_{i=0}^{d} A_{i} z^{-i}\right) \neq 0, \forall z \in \operatorname{ext}(\mathbb{D}) \cup \partial \mathbb{D} \text {. }
$$

- System (4) is asymptotically stable if and only if:

$$
\rho\left(A_{\xi}\right)<1 .
$$

Theorem 2. The following statements are equivalent:

- The delay difference equation (1) is asymptotically stable.

- The system (4) is asymptotically stable.

\section{D-INVARIANCE PROPERTIES}

Let us first consider the generic (nonlinear) discrete-time dynamical system:

$$
x(k+1)=f(x(k))
$$

where $x(k) \in \mathbb{R}^{n}$ is the state vector at time $k \in \mathbb{Z}_{+}$ and the function $f: \mathbb{R}^{n} \rightarrow \mathbb{R}^{n}$ is continuous.

Definition 6. The set $\mathcal{P} \subset \mathbb{R}^{n}$ is said positively invariant for the system (7) if for all $x(k) \in \mathcal{P}$, $x(k+1) \in \mathcal{P}$ for $k \in \mathbb{Z}_{+}$. Alternatively, the set $\mathcal{P} \subset \mathbb{R}^{n}$ is positively invariant for $(7)$ if $f(\mathcal{P}) \subseteq \mathcal{P}$.

Definition 7. Given a scalar $\epsilon \in \mathbb{R}_{(0,1)}$, a set $\mathcal{P} \subset$ $\mathbb{R}^{n}$ containing the origin is called $\epsilon$-contractive with respect to system (7) if for any $x(k) \in \mathcal{P}, x(k+1) \in$ $\epsilon \mathcal{P}$ for $k \in \mathbb{Z}_{+}$.

One can notice from Definitions 6 and 7 that positive invariance is a limit case of $\epsilon$-contractivness (it would amount to choosing $\epsilon=1$ in Definition 7 ). In the sequel, we will come back to these notions and detail analogies and particularities of time-delay systems. The $\mathcal{D}$-invariance concept, recalled below, will be widely used throughout this paper for the setcharacterization of dDDEs. The notations by [44, 45] will be mainly used in this endeavor.

Definition 8. A set $\mathcal{P} \subseteq \mathbb{R}^{n}$ is called $\mathcal{D}$-invariant for the system (1) with initial conditions $x_{-i} \in \mathcal{P}$ for all $i \in \mathbb{Z}_{[0, d]}$ if the state trajectory satisfies $x(k) \in$ $\mathcal{P}, \forall k \in \mathbb{Z}_{+}$.

Lemma 3. [52] The following statements are equivalent:

1. $\mathcal{P} \subseteq \mathbb{R}^{n}$ is $\mathcal{D}$-invariant for system (1).

2. $\bigoplus_{i=0}^{d} A_{i} \mathcal{P} \subset \mathcal{P}$ 
Several properties fix a set of basic relations between $\mathcal{D}$-invariant sets.

Proposition 1. The following properties hold:

1. If $\mathcal{P} \subset \mathbb{R}^{n}$ is $\mathcal{D}$-invariant then $\alpha \mathcal{P}$ is $\mathcal{D}$-invariant for any $\alpha \in \mathbb{R}_{+}$.

2. Let $\mathcal{P}_{1}, \mathcal{P}_{2} \subset \mathbb{R}^{n}$ be two $\mathcal{D}$-invariant sets for (1). Then $\mathcal{P}_{1} \cap \mathcal{P}_{2}$ is a $\mathcal{D}$-invariant set for the same dynamical system.

3. Let $\mathcal{P}_{1}, \mathcal{P}_{2} \subset \mathbb{R}^{n}$ be two $\mathcal{D}$-invariant sets for (1). The Minkowski sum $\mathcal{P}_{1} \oplus \mathcal{P}_{2}$ is a $\mathcal{D}$-invariant set for the same dynamical system.

4. If the set $\mathcal{P} \subset \mathbb{R}^{n}$ is $\mathcal{D}$-invariant for the system:

$$
x(k+1)=\sum_{i=0}^{d} A_{i} x(k-i)
$$

then $\mathcal{P}$ is $\mathcal{D}$-invariant for

$$
x(k+1)=\sum_{i=0}^{d} A_{i} x\left(k-\tau_{i}\right)
$$

for any $\tau_{i} \in \mathbb{Z}_{+}$.

5. If the compact set containing the origin $\mathcal{P}$ is $\mathcal{D}$ invariant, then its convex hull $\operatorname{Conv}(\mathcal{P})$ is $\mathcal{D}$ invariant.

6. If $\mathcal{P}_{1}, \mathcal{P}_{2} \subset \mathbb{R}^{n}$ are two $\mathcal{D}$-invariant sets for (1), their union $\mathcal{P}_{1} \cup \mathcal{P}_{2}$ is not necessarily $\mathcal{D}$ invariant.

7. The convex hull of the union of $\mathcal{D}$-invariant sets is not necessarily $\mathcal{D}$-invariant.

Proof. Properties (1), (2) and (4) were proved in [52]. The proof of properties (3) and (6) is straightforward. For the proof of property (5), one can exploit the relationship: $A_{1} \operatorname{Conv}(\mathcal{P}) \oplus A_{2} \operatorname{Conv}(\mathcal{P})=$ $\operatorname{Conv}\left(A_{1} \mathcal{P}\right) \oplus \operatorname{Conv}\left(A_{2} \mathcal{P}\right)=\operatorname{Conv}\left(A_{1} \mathcal{P} \oplus A_{2} \mathcal{P}\right)$. The first equality is a direct application of the convex hull definition and Minkowski sum properties. For the second equality, let $\mathcal{P}_{1}, \mathcal{P}_{2} \subset \mathbb{R}^{n}$, and let $x \in \operatorname{Conv}\left(\mathcal{P}_{1} \oplus \mathcal{P}_{2}\right)$, then $x=\sum \lambda_{i}\left(x_{i}+y_{i}\right)$ with $x_{i} \in \mathcal{P}_{1}$ and $y_{i} \in \mathcal{P}_{2}, \lambda_{i} \geq 0$ and $\sum \lambda_{i}=1$, then $x=\sum \lambda_{i} x_{i}+\sum \lambda_{i} y_{i} \in \operatorname{Conv}\left(\mathcal{P}_{1}\right) \oplus \operatorname{Conv}\left(\mathcal{P}_{2}\right)$. Suppose now that $x \in \operatorname{Conv}\left(\mathcal{P}_{1}\right) \oplus \operatorname{Conv}\left(\mathcal{P}_{2}\right)$ then $x=\sum \lambda_{i} x_{i}+\sum \beta_{j} y_{j}$, with $\sum \lambda_{i}=\sum \beta_{j}=1$, and $\lambda_{i}, \beta_{j} \geq 0, x_{i} \in \mathcal{P}_{1}, y_{j} \in \mathcal{P}_{2}$. since $\sum \lambda_{i} \sum \beta_{j}=$ $\sum_{i, j} \lambda_{i} \beta_{j}=1$ we can write $x=\sum_{i, j} \lambda_{i} \beta_{j}\left(x_{i}+y_{j}\right)$, then $x \in \operatorname{Conv}\left(\mathcal{P}_{1} \oplus \mathcal{P}_{2}\right)$. Note that

$$
A_{1} \mathcal{P} \oplus A_{2} \mathcal{P} \subset \mathcal{P} \Longrightarrow \operatorname{Conv}\left(A_{1} \mathcal{P} \oplus A_{2} \mathcal{P}\right) \subset \operatorname{Conv}(\mathcal{P})
$$

to conclude that:

$$
A_{1} \operatorname{Conv}(\mathcal{P}) \oplus A_{2} \operatorname{Conv}(\mathcal{P}) \subset \operatorname{Conv}(\mathcal{P})
$$

In order to check the property (7), consider the system:

$x(k+1)=\left[\begin{array}{cc}0.2 & 0.01 \\ 0 & 0.7\end{array}\right] x(k)+\left[\begin{array}{cc}0.6 & 0 \\ 0.005 & 0.25\end{array}\right] x(k-1)$,

then the set

$$
\mathcal{P}_{1}=\left\{x \in \mathbb{R}^{2} \mid\left[\begin{array}{c}
-0.1 \\
-1
\end{array}\right] \leq x \leq\left[\begin{array}{c}
0.1 \\
1
\end{array}\right]\right\}
$$

is $\mathcal{D}$-invariant as well as

$$
\mathcal{P}_{2}=\left\{x \in \mathbb{R}^{2} \mid\left[\begin{array}{c}
-1 \\
-0.1
\end{array}\right] \leq x \leq\left[\begin{array}{c}
1 \\
0.1
\end{array}\right]\right\}
$$

However, the set obtained as convex hull of the union $\mathcal{P}_{1} \cup \mathcal{P}_{2}$, denoted $\mathcal{P}=\operatorname{Conv}\left(\mathcal{P}_{1}, \mathcal{P}_{2}\right)$, is not $\mathcal{D}$-invariant.

Remark 1. Property (7) of Proposition 1 raises a warning on the convex hull operation applied to the union of two or more $\mathcal{D}$-invariant sets, which is not a closed operation over the class of $\mathcal{D}$-invariant sets. However, property (5) of Proposition 1 points out that for one $\mathcal{D}$-invariant operand, the convex hull operation preserves $\mathcal{D}$-invariance. It becomes clear that under the (unfortunately uncheckable) assumption that a $\mathcal{D}$-invariant set exists, an efficient (convexity based) construction will be able to characterize it.

Remark 2. The property (4) of Proposition 1 holds also for the limit case $\tau_{i}=\infty$. As a consequence, if $\mathcal{P} \subseteq \mathbb{R}^{n}$ is a $\mathcal{D}$-invariant set containing the origin, then $\mathcal{P}$ is positively invariant with respect to the time invariant linear dynamics:

$$
\begin{gathered}
x(k+1)=A_{0} x(k), \\
\vdots \\
x(k+1)=A_{d} x(k) .
\end{gathered}
$$

Equivalently, $A_{0} \mathcal{P} \subseteq \mathcal{P}, \cdots, A_{d} \mathcal{P} \subseteq \mathcal{P}$. The same result holds for a dDDE represented by a partial sum of (1). Note that the second property of Proposition 1 can be generalized. The intersection of a finite or infinite family of $\mathcal{D}$-invariant sets is $\mathcal{D}$-invariant. 
The goal of the next subsections is to collect necessary and/or sufficient conditions for the existence of a $\mathcal{D}$-invariant set for dDDEs. The existence of a nondegenerate and bounded $\mathcal{D}$-invariant set $^{1}$ is related to the stability of the discrete-time dynamical system (1) affected by delay. It is obvious that asymptotic stability is only a necessary condition for the existence of a $\mathcal{D}$-contractive set and stricter conditions have to be imposed for guaranteeing this existence. In the following we enumerate a series of necessary and/or sufficient conditions available in the literature, to the best of our knowledge; whenever possible, we will link the conditions to classical numerical routines for the eigenvalue problems.

\subsection{Necessary conditions for $\mathcal{D}$-invariance}

\subsubsection{Basic algebraic conditions}

Proposition 2. [44] Considering the system (1), the existence of a $\mathcal{D}$-invariant $\mathcal{C}$-set $\mathcal{P}$ implies that:

1. The spectral radii of the matrices $A_{i}$ are subunitary:

$$
\rho\left(A_{i}\right) \leq 1, \forall i \in \mathbb{Z}_{[0, d]}
$$

2. The spectral radius of the matrix $\left(\sum_{i=0}^{d} A_{i}\right)$ is sub-unitary:

$$
\rho\left(\sum_{i=0}^{d} A_{i}\right) \leq 1 .
$$

3. The spectral radius of the extended state-space matrix is sub-unitary:

$$
\rho\left(A_{\xi}\right) \leq 1
$$

Proposition 2 in conjunction with property (4) of Proposition 1 gives a measure of the complexity of establishing necessary and sufficient conditions. Practically, the difficulty is related to the need of testing the spectral radius of the extended state-space matrix for all possible delay realizations.

\subsubsection{Alternative algebraic conditions}

Alternative necessary conditions were proposed in [53] in terms of asymptotic stability of dDDEs, for the existence of a $\mathcal{D}$-contractive set. The main idea

\footnotetext{
${ }^{1}$ It is easy to observe that sets like $\{0\}$ or $\mathbb{R}^{n}$ are $\mathcal{D}$-invariant but they do not satisfy the non-degenerate or boundedness conditions.
}

is to cover the possible sign combinations for the tuple $A_{i}, i \in \mathbb{Z}_{[0, d]}$ : a straightforward task for any value of the delay parameter. In order to simplify the notation, let us introduce the set $\mathbb{S}=\{-1,0,1\}$ and $\Delta=[\delta(0), \cdots, \delta(d)]$.

Proposition 3. [53] System (1) admits a $\mathcal{D}$ contractive set only if:

$$
\rho\left(\sum_{i=0}^{d} \delta(i) A_{i}\right) \leq 1, \forall \Delta \in \mathbb{S}^{d+1} .
$$

If a given dDDE does not satisfy the above condition, then it does not admit a $\mathcal{D}$-contractive set. [53] shows that the condition derived in Proposition 3 is not sufficient for the existence of a $\mathcal{D}$-contractive set, numerical examples being available in this sense.

\subsubsection{Specific algebraic conditions for 2 delay dDDEs}

For dDDEs with two delay parameters, in order to decrease the conservativeness of the time-domain methods, [46] has used the frequency-domain framework. The $\mathcal{D}$-invariance concept was studied, along with its relation to robust asymptotic stability, considered as a strong stability of dDDEs. This notion defines stability with respect to all delay realizations. Due to the incompleteness of the discrete time, the characterization of robust asymptotic stability is not simple. Thus using a more general class of difference equation (precisely the ones that are specified in the continuous-time domain) proved to be useful. In the sequel the concept of strong stability is denoted by delay-independent stability ${ }^{2}$ and it represents the continuous-time counterpart to robust asymptotic stability.

Recently, [46] has provided a computationally efficient numerical condition which is necessary to guarantee the existence of Lyapunov-Razumikhin contractive sets. This test is sufficient for the robust asymptotic stability with respect to the delay parameter and can be employed in the $\mathcal{D}$-invariance context. The main result can be summarized in the next theorem.

Theorem 4. [46] Assume that $\rho\left(A_{0}+A_{1}\right) \leq 1$ and that $d_{0} \in \mathbb{R}_{+}$and $d_{1} \in \mathbb{R}_{+}$. Then, the system

$$
x(k)=\sum_{i=0}^{1} A_{i} x\left(k-d_{i}\right)
$$

\footnotetext{
${ }^{2}$ also known as stability in the delays.
} 
admits a $\mathcal{D}$-contractive set only if $\gamma(U, V) \cap \partial \mathbb{D}=\emptyset$, where

$$
\begin{array}{r}
U=\left(\begin{array}{cc}
0_{n^{2} \times n^{2}} & I_{n^{2}} \\
-B_{0} & -B_{1}
\end{array}\right), V=\left(\begin{array}{cc}
I_{n^{2}} & 0_{n^{2} \times n^{2}} \\
0_{n^{2} \times n^{2}} & B_{2}
\end{array}\right) \\
B_{0}=A_{0} \otimes A_{1}^{T}, B_{1}=A_{0} \otimes A_{0}^{T}+A_{1} \otimes A_{1}^{T}-I_{n^{2}} \\
B_{2}=A_{1} \otimes A_{0}^{T}
\end{array}
$$

As stated in [46], the condition of Theorem 4 covers the existing necessary conditions for the two delay parameters case. However, we report here an interesting example which points out the possible limitations of this condition.

Example 1. [39] Consider system (1) with $d=1$ and:

$$
A_{0}=\left(\begin{array}{cc}
0.5 & 0.5 \\
0 & 0
\end{array}\right) ; \quad A_{1}=\left(\begin{array}{cc}
0 & 0.5 \\
-0.5 & 0.5
\end{array}\right)
$$

For this numerical example, one can compute:

$$
\rho\left(A_{0}+A_{1}\right)=0.8660<1
$$

and

$\gamma(U, V)=1.7442 \pm 1.9433 \mathrm{i}, 0.2558 \pm 0.2850 \mathrm{i}, 0,0$, inf, inf.

The necessary condition by [46] is fulfilled. However, up to the existing constructive routines (see next section) there is no numerical construction able to determine a $\mathcal{D}$-invariant set for this system.

\subsection{Sufficient conditions for $\mathcal{D}$-invariance}

The converse problem of establishing sufficient conditions for the existence of $\mathcal{D}$-invariant sets has been stated in [52] with two tests that we recall here for completeness.

Proposition 4. [52] The existence of a $\mathcal{D}$-invariant $\mathcal{C}$-set $\mathcal{P}$ is guaranteed for the system (1), if one of the following spectral norm based conditions holds:

1. The sum of the spectral norms of $A_{i}$, for $i \in$ $\mathbb{Z}_{[0, d]}$, is subunitary:

$$
\sum_{i=0}^{d} \sigma\left(A_{i}\right)<1 .
$$

2. In the case of nonsingular matrix $A_{i}$ for $i \in \mathbb{Z}_{[0, d]}$

$$
\begin{gathered}
\left(1+\sigma\left(A_{0}^{-1} A_{1}\right)+\cdots+\sigma\left(A_{0}^{-1} A_{d}\right)\right) \sigma\left(A_{0}\right) \leq 1 \\
\vdots \\
\left(1+\sigma\left(A_{d}^{-1} A_{0}\right)+\cdots+\sigma\left(A_{d}^{-1} A_{d-1}\right)\right) \sigma\left(A_{d}\right) \leq 1 .
\end{gathered}
$$

Remark 3. The sufficient condition (1) can be generalized by replacing the sum of the spectral norms by the sum of any other induced matrix norms.

Proposition 4 concentrates on the spectral norms of the matrices appearing in the dDDE (1). A different approach for establishing sufficient conditions is to exploit the structural properties of specific classes of candidate $\mathcal{D}$-invariant sets. We propose next a contribution in this sense with a sufficient condition for the existence of ellipsoidal $\mathcal{D}$-contractive sets for a dDDE. As it is often the case in this framework, the tests are based on LMIs.

Theorem 5. Considering the dynamical system (1), the existence of an ellipsoidal $\mathcal{D}$-invariant set is guaranteed if the following $d+1$ LMIs hold for some $P=P^{T} \succ 0$ :

$$
\begin{aligned}
& \left(\begin{array}{cccc}
A_{0}^{T} P A_{0}-P & A_{0}^{T} P A_{1} & \cdots & A_{0}^{T} P A_{d} \\
A_{1}^{T} P A_{0} & A_{1}^{T} P A_{1} & \cdots & A_{1}^{T} P A_{d} \\
\vdots & \vdots & \vdots & \vdots \\
A_{d}^{T} P A_{0} & A_{d}^{T} P A_{1} & \cdots & A_{d}^{T} P A_{d}
\end{array}\right) \prec 0 \\
& \left(\begin{array}{cccc}
A_{0}^{T} P A_{0} & A_{0}^{T} P A_{1} & \cdots & A_{0}^{T} P A_{d} \\
A_{1}^{T} P A_{0} & A_{1}^{T} P A_{1}-P & \cdots & A_{1}^{T} P A_{d} \\
\vdots & \vdots & \vdots & \vdots \\
A_{d}^{T} P A_{0} & A_{d}^{T} P A_{1} & \cdots & A_{d}^{T} P A_{d}
\end{array}\right) \prec 0 \\
& \left(\begin{array}{cccc}
A_{0}^{T} P A_{0} & A_{0}^{T} P A_{1} & \cdots & A_{0}^{T} P A_{d} \\
A_{1}^{T} P A_{0} & A_{1}^{T} P A_{1} & \cdots & A_{1}^{T} P A_{d} \\
\vdots & \vdots & \vdots & \vdots \\
A_{d}^{T} P A_{0} & A_{d}^{T} P A_{1} & \cdots & A_{d}^{T} P A_{d}-P
\end{array}\right) \prec 0
\end{aligned}
$$

PRoof. In order to ensure that the set

$$
\Psi=\left\{x \in \mathbb{R}^{n}, x^{T} P x \leq 1\right\}
$$

is $\mathcal{D}$-invariant for the system described by the dDDE (1), one has to show that $x_{k+1} \in \Psi$, 
$\forall x_{k}, x_{k-1}, \cdots, x_{k-d} \in \Psi$, which is equivalent to the simultaneous verification of the $d+1$ inequalities:

$$
\begin{gathered}
x_{k+1}^{T} P x_{k+1}-x_{k}^{T} P x_{k}<0 \\
x_{k+1}^{T} P x_{k+1}-x_{k-1}^{T} P x_{k-1}<0 \\
\vdots \\
x_{k+1}^{T} P x_{k+1}-x_{k-d}^{T} P x_{k-d}<0
\end{gathered}
$$

Exploiting the dDDE relationship one has: $x_{k+1}^{T} P x_{k+1}-x_{k}^{T} P x_{k}=\left(A_{0} x_{k}+A_{1} x_{k-1}+\cdots+\right.$ $\left.A_{d} x_{k-d}\right)^{T} P\left(A_{0} x_{k}+A_{1} x_{k-1}+\cdots+A_{d} x_{k-d}\right)-x_{k}^{T} P x_{k}$ $=x_{k}^{T}\left(A_{0}^{T} P A_{0}-P\right) x_{k}+x_{k}^{T} A_{0}^{T} P\left(A_{1} x_{k-1}+\cdots+\right.$ $\left.A_{d} x_{k-d}\right)+\left(A_{1} x_{k-1}+\cdots+A_{d} x_{k-d}\right)^{T} P\left(A_{0} x_{k}+\right.$ $\left.A_{1} x_{k-1}+\cdots+A_{d} x_{k-d}\right)<0$.

and in the equivalent matrix formulation:

$$
\left(\begin{array}{c}
x_{k} \\
x_{k-1} \\
\vdots \\
x_{k-d}
\end{array}\right)^{T}\left(\begin{array}{cccc}
A_{0}^{T} P A_{0}-P & A_{0}^{T} P A_{1} & \cdots & A_{0}^{T} P A_{d} \\
A_{1}^{T} P A_{0} & A_{1}^{T} P A_{1} & \cdots & A_{1}^{T} P A_{d} \\
\vdots & \vdots & \vdots & \vdots \\
A_{d}^{T} P A_{0} & A_{d}^{T} P A_{1} & \cdots & A_{d}^{T} P A_{d}
\end{array}\right)\left(\begin{array}{c}
x_{k} \\
x_{k-1} \\
\vdots \\
x_{k-d}
\end{array}\right) \prec 0
$$

Analogously for the second inequality:

$\left(\begin{array}{c}x_{k} \\ x_{k-1} \\ \vdots \\ x_{k-d}\end{array}\right)^{T}\left(\begin{array}{cccc}A_{0}^{T} P A_{0} & A_{0}^{T} P A_{1} & \cdots & A_{0}^{T} P A_{d} \\ A_{1}^{T} P A_{0} & A_{1}^{T} P A_{1}-P & \cdots & A_{1}^{T} P A_{d} \\ \vdots & \vdots & \vdots & \vdots \\ A_{d}^{T} P A_{0} & A_{d}^{T} P A_{1} & \cdots & A_{d}^{T} P A_{d}\end{array}\right)\left(\begin{array}{c}x_{k} \\ x_{k-1} \\ \vdots \\ x_{k-d}\end{array}\right) \prec 0$

up to the $d+1$ inequality. We can conclude that the existence of a positive definite matrix $P=P^{T}$ is a sufficient condition for the existence of an ellipsoidal $\mathcal{D}$-invariant set, and the proof is complete.

Example 2. For illustration let us consider system (1) with only one delay parameter $d=1$ and:

$$
A_{0}=\left(\begin{array}{cc}
0.35 & 0.13 \\
0.51 & -0.01
\end{array}\right), A_{1}=\left(\begin{array}{cc}
0.51 & -0.01 \\
0.03 & 0.51
\end{array}\right)
$$

The condition for the existence of a $\mathcal{D}$-contractive set proposed in Theorem 5 is fulfilled and the $\mathcal{D}$ contractive set exists as shown in Figure 1. Dashed black lines in Figure 1 represent the state trajectories starting from some points on the boundary of the ellipsoidal $\mathcal{D}$-contractive set with respect to the dDDE (1) with $d=1, A_{0}, A_{1}$ given in (22). It is interesting to note that the sufficient condition $\left\|A_{0}\right\|_{p}+\left\|A_{1}\right\|_{p} \leq 1$ by $[42,44]$ does not hold for this numerical example.

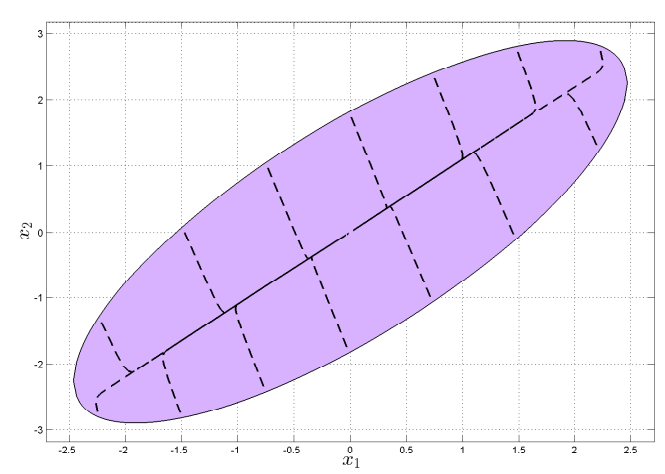

Figure 1: $\mathcal{D}$-contractive set for the $\operatorname{dDDE}(1)$ with $d=1$, $A_{0}, A_{1}$ given in (22).

\subsection{Necessary and sufficient algebraic conditions for Polyhedral D-invariant sets}

The problem of finding convex $\mathcal{D}$-invariant sets can benefit whenever particular structural properties are $\prec 0$ enforced. It is the case of polyhedral sets, for which necessary and sufficient conditions exist as resumed by the following theorem.

Theorem 6. [54] Let a delay difference equation be described by (1). There exists $\mathcal{P}$ a polyhedral $\mathcal{D}$ contractive set containing the origin:

$$
\mathcal{P}=\left\{x \in \mathbb{R}^{n} \mid F x \leq \mathbf{1}\right\}
$$

with $F \in \mathbb{R}^{r \times n}$, described by its minimal half space representation, if and only if there exist $d+1$ real matrices $H_{i} \in \mathbb{R}^{r \times r}$, for $i=\{0, \cdots, d\}$, with nonnegative elements and a positive $\epsilon<1$, such that:

$$
F A_{i}=H_{i} F
$$

$$
\left(\sum_{i=0}^{d} H_{i}\right) \mathbf{1}_{r} \leq \epsilon \mathbf{1}_{r}
$$

Clearly, if the requirement on $\epsilon$ being strictly smaller than 1 is relaxed to non-strict inequality, then (24) represents a necessary and sufficient condition for the existence of a $\mathcal{D}$-invariant set.

\subsection{Relationship between $\mathcal{D}$-invariance and $d D D E$ stability}

In this subsection we aim at complementing the overview of the necessary and sufficient conditions with a theoretical result that establishes a link between the stability in presence of time-varying delay and the existence of $\mathcal{D}$-invariant sets. 
Theorem 7. The dDDE (2) admits a proper $\mathcal{D}$ invariant set if and only if the time-varying $d D D E$ (3) is delay-independent stable.

Proof. We prove next the case of dDDE with only two delay parameters, $x(k+1)=A_{0} x\left(k-d_{0}\right)+$ $A_{1} x\left(k-d_{1}\right)$, the case of finite number of delays (2) being a direct generalization. The proof of the "only if" implication builds on the fact that the existence of a $\mathcal{D}$-invariant set $\mathcal{P}$ is equivalent with the set inclusion:

$$
A_{0} \mathcal{P} \oplus A_{1} \mathcal{P} \subset \mathcal{P}
$$

Thus for initial conditions $x(k) \in \mathcal{P}$ for $k \in \mathbb{Z}_{(-\infty, 0]}$ one has $x(1) \in \mathcal{P}$ independent of the delay realization $d_{0}(0), d_{1}(0) \in \mathbb{N}$. By induction, given a positive index $i \in \mathbb{N}$, if $x(k) \in \mathcal{P}$ for $k \in \mathbb{Z}_{(-\infty, i]}$ then $x(i+1) \in \mathcal{P}$ independent of the delay realization $d_{0}(i), d_{1}(i) \in \mathbb{N}$ which implies that the trajectories are bounded $x(k) \in \mathcal{P}, \forall k \in \mathbb{N}_{+}$. Stability for any initial condition follows from property (1) of Proposition 1. By homogeneity, $\mathcal{D}$-invariance is preserved by scaling and as such, there always exists a $\mathcal{D}$-invariant set which contains a given initial condition of the dDDE.

For the "if" part of the proof, consider the initial conditions for the system (3) to be contained in a compact set $\mathcal{P}$ containing the origin in its interior. Formally, the initial conditions and the time-varying delay realization can be described by the functions:

$$
\begin{aligned}
& \mathbf{x}_{\mathcal{P}}^{-}: \mathbb{Z}_{(-\infty, 0]} \rightarrow \mathcal{P} \\
& \mathbf{d}_{\mathbf{0}}: \mathbb{N}_{+} \rightarrow \mathbb{Z}_{(-\infty, 0]} \\
& \mathbf{d}_{\mathbf{1}}: \mathbb{N}_{+} \rightarrow \mathbb{Z}_{(-\infty, 0]}
\end{aligned}
$$

Having as an objective the construction of the reachable set from $\mathcal{P}$, let us denote the state at time instant $k \in \mathbb{Z}$ by $x\left(k, \mathbf{x}_{\mathcal{P}}^{-}, \mathbf{d}_{\mathbf{0}}, \mathbf{d}_{\mathbf{1}}\right)$ as the solution of (3) with respect to the initial conditions $\mathbf{x}_{\mathcal{P}}^{-}$and time-varying delay realizations $\mathbf{d}_{\mathbf{0}}(\cdot), \mathbf{d}_{\mathbf{1}}(\cdot)$. With this notation, the reachable set from $\mathcal{P}$ via $(3)$ is defined as:

$$
\begin{gathered}
\mathcal{R}(\mathcal{P})=\left\{x \in \mathbb{R}^{n} \mid \exists k \in \mathbb{N}_{+}, \mathbf{x}_{\mathcal{P}}^{-}(\cdot), \mathbf{d}_{\mathbf{0}}(\cdot), \mathbf{d}_{\mathbf{1}}(\cdot)\right. \text { s.t. } \\
\left.x=x\left(k, \mathbf{x}_{\mathcal{P}}^{-}, \mathbf{d}_{\mathbf{0}}, \mathbf{d}_{\mathbf{1}}\right)\right\}
\end{gathered}
$$

Coming back to the proof, the objective is to show that $\mathcal{P}_{r}=\mathcal{P} \cup \mathcal{R}(\mathcal{P})$ is a proper $\mathcal{D}$-invariant set. The fact that the origin is contained in the interior of $\mathcal{P}_{r}$ is inherited from the properties of $\mathcal{P}$. The boundedness of the set $\mathcal{R}(\mathcal{P})$ is ensured by the stability assumption and will be inherited by $\mathcal{P}_{r}$. What remains to be proved is the invariance of $\mathcal{P}_{r}$. Three possibilities should be discussed:
- $x\left(k-d_{0}(k)\right) \in \mathcal{P}$ and $x\left(k-d_{1}(k)\right) \in \mathcal{P}$ : in this case the state $x(k+1)$ is part of the one step reachable set and subsequently $x(k+1) \in$ $\mathcal{R}(\mathcal{P}) \subset \mathcal{P}_{r}$

- $x\left(k-d_{0}(k)\right) \in \mathcal{P}$ and $x\left(k-d_{1}(k)\right) \in \mathcal{R}(\mathcal{P})$ (with delay indices which can be interchanged): this case corresponds to a reachable state $x(k-$ $\left.d_{1}(k)\right) \in \mathcal{R}(\mathcal{P})$ combined with a large (pseudoinfinite) delay $d_{0}(k)$. By consequence the state realizations $x(k+1)$ will represent a subset of the reachable set and $\mathcal{R}(\mathcal{P}) \subset \mathcal{P}_{r}$.

- $x\left(k-d_{0}(k)\right) \in \mathcal{R}(\mathcal{P})$ and $x\left(k-d_{1}(k)\right) \in \mathcal{R}(\mathcal{P})$ (with delay indices which can be interchanged): again, via reachability $x(k+1) \in \mathcal{R}(\mathcal{P}) \subset \mathcal{P}_{r}$ with the particular case $d_{0}(k)=d_{1}(k)$ which deserves a special treatment. Indeed, for the restriction $d_{0}(k)=d_{1}(k)$, the state dynamics $(3)$ reduces to $x(k+1)=\left(A_{0}+A_{1}\right) x\left(k-d_{1}(k)\right)$. But this realization is only a particular case of the general time-varying delay realization $d_{0}(k) \neq$ $d_{1}(k)$ for which $x\left(k-d_{0}(k)\right)=x\left(k-d_{1}(k)\right)$ which is covered by the reachable set construction and the proof is complete.

Remark 4. The sets containing the forward trajectories, as those used in the argument of the proof, are non-convex and lead to computationally demanding constructions, from a practical point of view. In the next section we describe the corresponding algorithm and subsequently reinforce the convexity by exploiting property (5) of Proposition 1.

\section{Construction of $\mathcal{D}$-invariant sets based on set iterations}

We address now the construction procedures for the case $x(k+1)=A_{0} x(k)+A_{d} x(k-d)$ supposing that it admits a $\mathcal{D}$-invariant set. The general form (1) follows similarly. We use the fact that existence of $\mathcal{D}$ invariant sets is exactly equivalent, by Lemma (3), to the verification of $A_{0} \mathcal{P} \oplus A_{d} \mathcal{P} \subseteq \mathcal{P}$. To simplify the explanation, we first define the forward mapping :

$$
\begin{aligned}
\Phi: \quad & \operatorname{Com}\left(\mathbb{R}^{n}\right) \rightarrow \operatorname{Com}\left(\mathbb{R}^{n}\right) \\
& \Phi(\mathcal{P})=A_{0} \mathcal{P} \oplus A_{d} \mathcal{P}
\end{aligned}
$$

and the mapping based on the union:

$$
\begin{aligned}
\Psi: \quad & \operatorname{Com}\left(\mathbb{R}^{n}\right) \rightarrow \operatorname{Com}\left(\mathbb{R}^{n}\right) \\
& \Psi(\mathcal{P})=\bigcup(\mathcal{P}, \Phi(\mathcal{P})) .
\end{aligned}
$$


Note that even if $\mathcal{P}$ is convex, $\Psi(\mathcal{P})$ is not necessarily convex.

Remark 5. We enumerate here some useful properties of the mappings defined in (29-30):

1. If a given set $\mathcal{P}$ (convex or not) is $\mathcal{D}$-invariant for $(1)$, then $\Phi(\mathcal{P}) \subseteq \mathcal{P}$.

2. k-iterates over the family of sets is set-wise non decreasing $\left(\Psi^{k-1}(\mathcal{P}) \subseteq \Psi^{k}(\mathcal{P}), \forall k \geq 1\right)$ with $\Psi^{k}(\mathcal{P})=\Psi\left(\Psi^{k-1}(\mathcal{P})\right)$ for $k>0$ and $\Psi^{0}(\mathcal{P})=\mathcal{P}$.

3. If $\mathcal{P}$ is $\mathcal{D}$-invariant for $(1)$ then $\Phi^{k}(\mathcal{P})$ is set-wise non increasing $\left(\Phi^{k}(\mathcal{P}) \subseteq \Phi^{k-1}(\mathcal{P}), \forall k \geq 1\right)$.

\subsection{Basic set-iterates procedure for the construction of $\mathcal{D}$-invariant sets}

We describe in this part the basic steps of an iterative construction of $\mathcal{D}$-invariant sets. Under the assumption that such an invariant set exists for the system (1), we can always scale it using property (1) of Proposition (1) such that it encompasses the initial set $\mathcal{Q}$. Using the theoretical properties shown above, an algorithmic routine based on non-convex sets mapping is proposed for the computation of $\mathcal{D}$-invariant sets with respect to (1). This algorithm considers as an input argument an arbitrary bounded set $\mathcal{Q}$ containing the origin $([55,56])$.
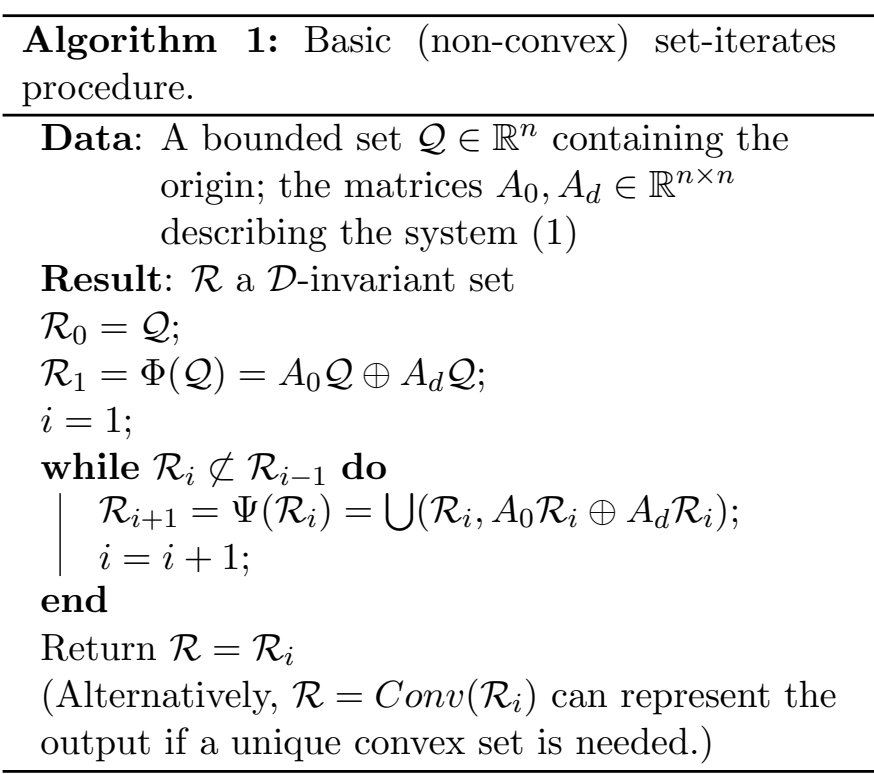

Convergence and finite determinedness analysis: First, it can be proved that Algorithm 1 constructs a non-decreasing sequence that converges to a $\mathcal{D}$-invariant set. Indeed, the algorithm is based on the set mapping $\mathcal{R}_{i+1}=\Psi\left(\mathcal{R}_{i}\right)$ which satisfies $\mathcal{R}_{i+1} \supset \mathcal{R}_{i}$. Thus the sequence $\mathcal{R}_{i}$ is non-decreasing in the sense of set inclusion. On the other hand, since the $\mathcal{D}$-invariance is scalable (using property (1) of Proposition (1)), the hypothesis of existence of a $\mathcal{D}$-invariant set $\mathcal{P}$ containing $\mathcal{Q}$ ensures $\mathcal{Q} \subset \mathcal{R}_{i} \subset \mathcal{P}$. Since any set $\mathcal{R}_{i}$ provided by the algorithm is a subset of $\mathcal{P}$, hence $\Psi\left(\mathcal{R}_{i}\right)$ is also a subset of $\mathcal{P}$. In conclusion, the algorithm provides a sequence of sets $\mathcal{R}_{i}$ which is non-decreasing by inclusion and limited from above by $\mathcal{P}$. Hence the sequence admits a limit which is $\mathcal{D}$-invariant (by the structure of the algorithm) and proper (because limited from above by $\mathcal{P}$ which is a fixed point with respect to the mapping $\Psi(\cdot))$. Secondly, the finite determinedness can be formally proved. Given the (delay-independent) asymptotic stability of system (1) with matrices $A_{0}$ and $A_{d}$, there exists a finite number of time steps $t_{\max }$ such that the trajectories initiated in $\mathcal{Q}$ end up in $\mathcal{P}$. The algorithm is collecting the trajectories initiated in $\mathcal{Q}$, which is a subset of $\mathcal{P}$, and thus $t_{\max }$ represents an upper bound for the number of iterations. This completes the convergence analysis of the algorithm.

Note that the iterations and the limit set are nonconvex and this is related to the union operation performed by the mapping in $\Psi(\cdot)$.

Example 3. [39] Let us consider the following dynamical system:

$x(k+1)=\left[\begin{array}{cc}0.1 & 0 \\ 0.4 & 0.1\end{array}\right] x(k)+\left[\begin{array}{cc}0.1 & -0.2 \\ 0.4 & 0.5\end{array}\right] x(k-d)$,

Consider the initialization set $\mathcal{Q}$ as the $\infty$-norm unit ball in $\mathbb{R}^{2}$. A non-convex $\mathcal{D}$-invariant set is obtained iteratively by applying Algorithm 1 with 4 iterations.
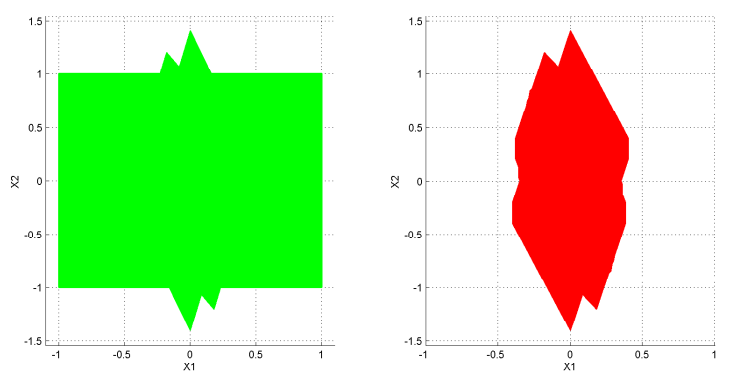

Figure 2: Graphical illustration of the non convex $\mathcal{D}$-invariant set for the Example 3 . The $\mathcal{D}$-invariant set-green (left); the set $A_{0} \mathcal{P} \oplus A_{d} \mathcal{P}$-red (right).

Figure 2 presents this invariant set (the left one), and the image (the right one) of this set by the forward mapping $\Phi(\cdot)$. Figure 3 presents the Convex 
hull of the obtained non-convex $\mathcal{D}$-invariant set and shows that it is $\mathcal{D}$-invariant as theoretically proved in property (5) of Proposition 1.

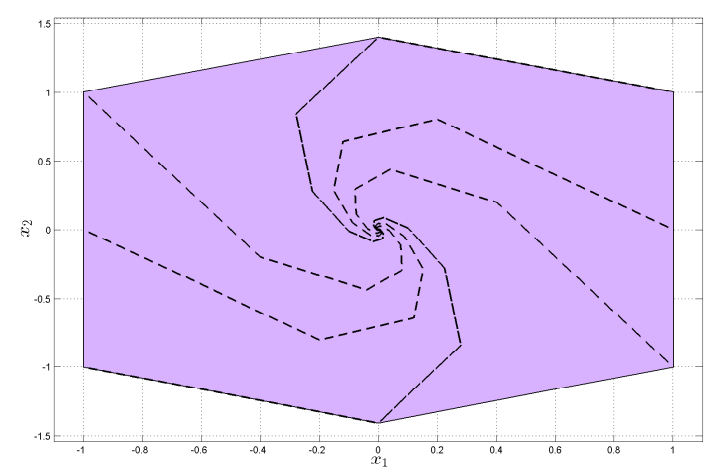

Figure 3: Graphical illustration of the convex $\mathcal{D}$-invariant set for the Example 3.

\subsection{Convex set-iterates procedure for the construc-} tion of $\mathcal{D}$-invariant sets

We describe briefly in this part the main steps of an iterative construction of $\mathcal{D}$-invariant sets while manipulating only convex sets. This algorithmic routine was proposed by [44], but we recall it here in light of Theorem 7 and Algorithm 1. Let us define the two mappings :

$$
\begin{array}{ll}
\Omega: \quad & \operatorname{Com} C\left(\mathbb{R}^{n}\right) \rightarrow \operatorname{Com} C\left(\mathbb{R}^{n}\right) \\
& \Omega(\mathcal{P})=A_{0} \mathcal{P} \oplus A_{d} \mathcal{P} \\
& \\
\Xi: \quad \operatorname{ComC}\left(\mathbb{R}^{n}\right) \rightarrow \operatorname{Com} C\left(\mathbb{R}^{n}\right) \\
& \Xi(\mathcal{P})=\operatorname{Conv}(\mathcal{P}, \Omega(\mathcal{P})) .
\end{array}
$$

Given a convex set $\mathcal{P} \in \operatorname{Com} C\left(\mathcal{R}^{n}\right)$, the sequence $\Xi^{k}(\mathcal{P}), k>0$ converges toward a convex $\mathcal{D}$-invariant set [44]. The main objective of this procedure remains the same as the previous one: enlarge the set as much as possible with the Convex hull operation, while keeping it included in a $\mathcal{D}$-invariant superset.

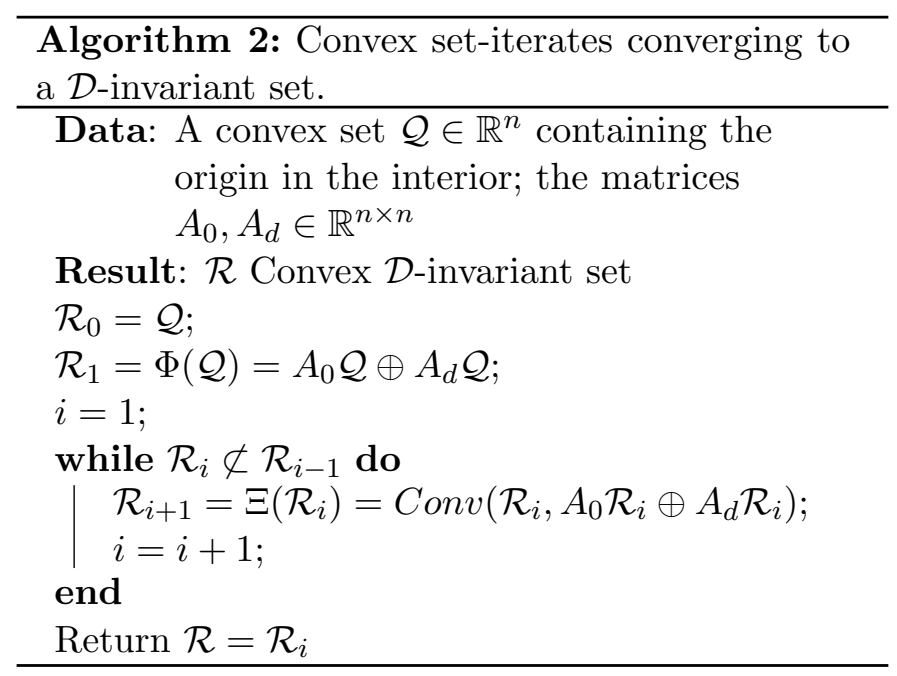

This algorithm, unlike the previous one, manipulates convex sets with all their computational advantages. At each iteration, the convex hull of the union of the present set and the forward mapping of the same set $\mathcal{R}_{i}$ are obtained.

\subsection{Complexity and speed of convergence}

In this section, we point to the possible extension of Algorithms 1-2 in order to improve the convergence speed. Instead of performing one forward mapping in each iteration before checking $\mathcal{D}$-invariance, $\mathrm{N}$ forward mappings are performed in each iteration. This seems to be efficient in the sense that we can reduce the complexity and the number of iterations.

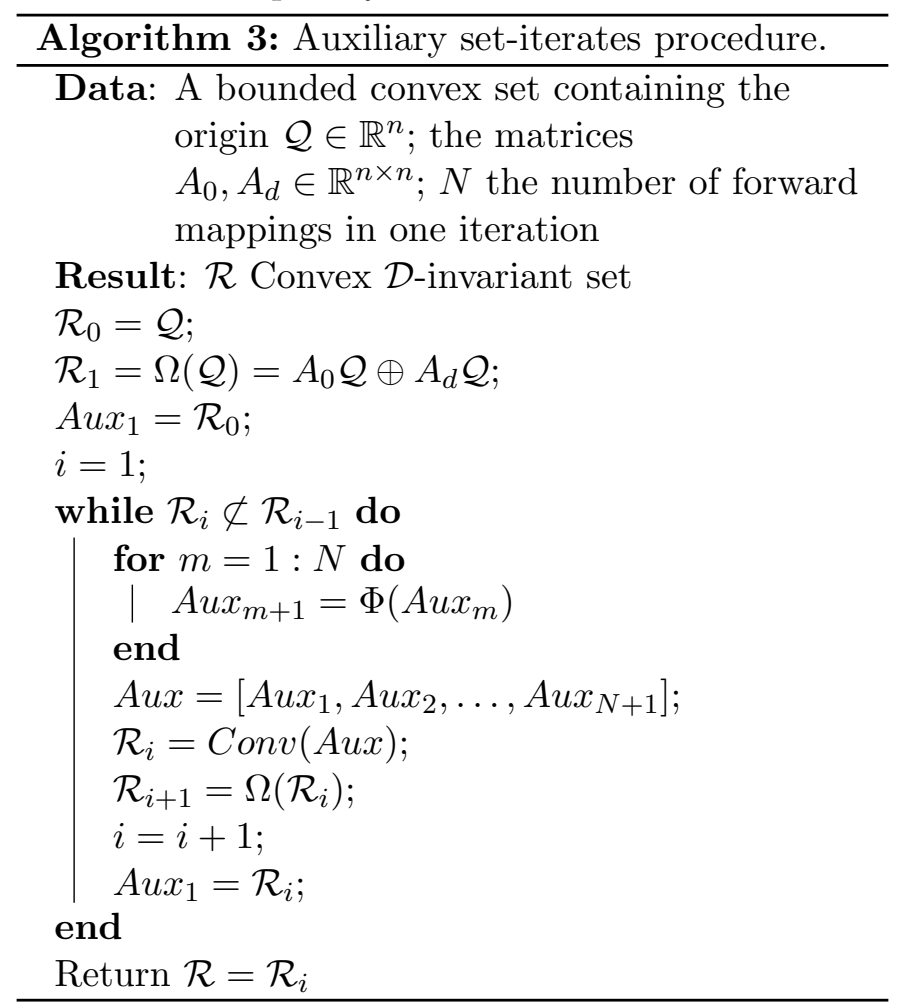


Example 4. [39] Let us consider the following dynamical system :

$$
x(k+1)=\left[\begin{array}{cc}
0.2 & 0.1 \\
0 & 0.6
\end{array}\right] x(k)+\left[\begin{array}{cc}
0.5 & 0 \\
0.1 & 0.3
\end{array}\right] x(k-d) .
$$

Let

$$
\mathcal{Q}=\left\{x \in \mathbb{R}^{2} \mid\left[\begin{array}{cc}
\sqrt{2} & -\sqrt{2} \\
-\sqrt{2} & \sqrt{2} \\
\sqrt{2} & \sqrt{2} \\
-\sqrt{2} & -\sqrt{2}
\end{array}\right] x \leq\left[\begin{array}{c}
0.5 \\
1 \\
0.5 \\
1
\end{array}\right]\right\}
$$

be the initialization set. By applying Algorithm 3 with $N=2$ and Algorithm 2, two different $\mathcal{D}$ invariant sets are obtained for the dynamical system (34) in $2 *(N=2)$ and 18 iterations, respectively. Figure 4 presents these sets. Dashed black lines represent the state trajectories starting from the vertices of these sets with respect to the dynamics (34).
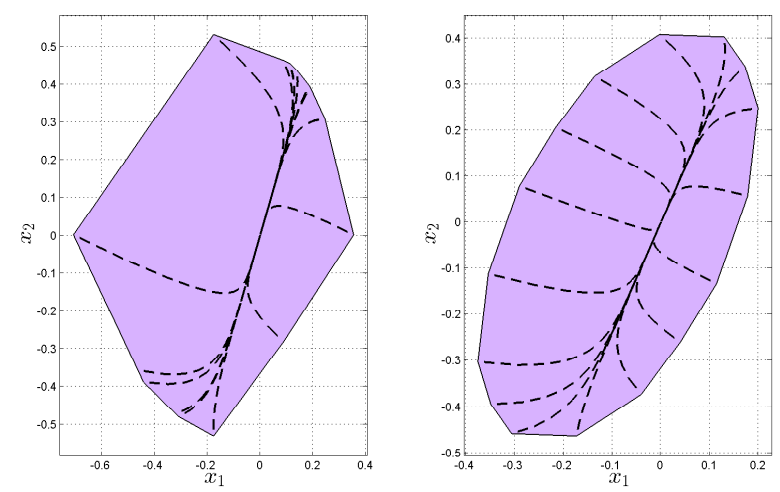

Figure 4: Graphical illustration of $\mathcal{D}$-invariant sets obtained by Algorithm 2 (left) and Algorithm 3 (right), for the Example 4.

It becomes clear that, under the assumption that a $\mathcal{D}$-invariant set exists, an efficient construction exists. We can also use the algorithmic construction (Algorithm 2) as an induced tool to check if a $\mathcal{D}$ invariant set can/cannot be obtained, whenever the dDDE satisfies the necessary conditions for the existence of such invariant sets. To illustrate this idea, Example 1, which raises a doubt about the sufficiency of the matrix-pencil based conditions [46], will be discussed in the sequel. By computing the set iterations up to strict inclusion into the initial one, convergence/divergence can be inferred. If the initial set $\mathcal{Q}$ for Algorithm 2 is the $\infty$-norm unit ball in $\mathbb{R}^{2}$ and the $\mathrm{dDDE}$ is given by the matrices in Example 1, then after 4 iterations one obtains the sequence in Figure
5. The set iteration can be stopped as long as $\mathcal{Q}$ is a strict subset of $\mathcal{P}_{4}$. This represents a proof by construction that forward set iterations diverge and the system does not admit a $\mathcal{D}$-invariant set.

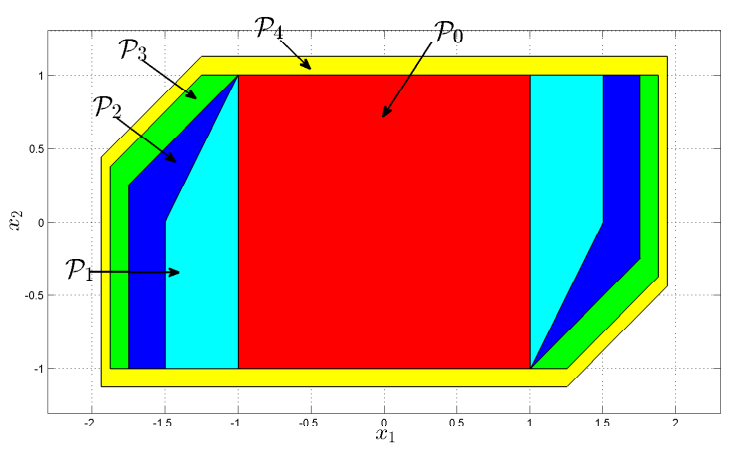

Figure 5: Sequence of the forward mappings $\operatorname{Conv}\left(\mathcal{P}, A_{0} \mathcal{P} \oplus\right.$ $\left.A_{d} \mathcal{P}\right)$, for the Example 1.

A pictorial overview of the relation between different kinds of stability and existence of $\mathcal{D}$-invariant sets is given in Figure 6. Solid black lines represent implications that have been proved herein. Solid yellow lines represent previous results and dashed lines with question marks represent open problems. Dashed lines with a cross between two statements show that the first property does not necessarily imply the second.

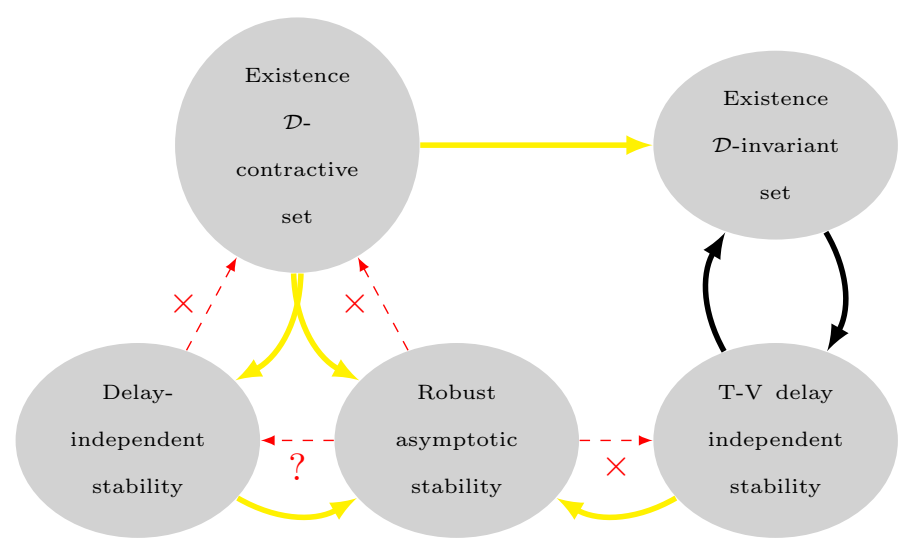

Figure 6: Schematic overview of the presented results.

\section{Extensions of $\mathcal{D}$-invariance}

As mentioned in the introduction, two main approaches exist in the literature dealing with positive invariant sets for discrete time-delay difference equations; an invariant set for the dDDE can be computed 
either in an extended state space, or in the original state space (in this latter case, it is called $\mathcal{D}$-invariant set). The concept of cyclic invariance [37] can be exploited to compute, instead of a rigid set in $\left(\mathbb{R}^{n}\right)^{d+1}$ or $\mathbb{R}^{n}$ as in the two aforementioned approaches, a tuple of invariant sets; thus offering a certain degree of flexibility.

Definition 9. A $(d+1)$-tuple of sets $\left\{\Omega_{0}, \ldots, \Omega_{d}\right\}$ is called cyclic $\mathcal{D}$-invariant with respect to (1) if:

$$
\begin{gathered}
A_{0} \Omega_{0} \oplus A_{1} \Omega_{1} \oplus \cdots \oplus A_{d} \Omega_{d} \subseteq \Omega_{d} \\
A_{0} \Omega_{d} \oplus A_{1} \Omega_{0} \oplus \cdots \oplus A_{d} \Omega_{d-1} \subseteq \Omega_{d-1} \\
\vdots \\
A_{0} \Omega_{1} \oplus A_{1} \Omega_{2} \oplus \cdots \oplus A_{d} \Omega_{0} \subseteq \Omega_{0}
\end{gathered}
$$

A generalization of the cyclic invariance notion to invariant family of sets was proposed by $[38,36]$.

Definition 10. A family of $(d+1)$-tuples of sets $\mathcal{F} \subset\left(\mathbb{R}^{n}\right)^{d+1}$ is an invariant family with respect to (1) if for any tuple $\left\{\Omega_{0}, \Omega_{1}, \ldots, \Omega_{d}\right\} \in \mathcal{F}$ there exists a set $\Omega_{*} \subset \mathbb{R}^{n}$ such that $\left\{\Omega_{*}, \Omega_{0} \ldots, \Omega_{d-1}\right\} \in \mathcal{F}$ and $\mathrm{A}_{0} \Omega_{0} \oplus A_{1} \Omega_{1} \oplus \cdots \oplus A_{d} \Omega_{d} \subseteq \Omega_{*}$.

The link between the two main representations for discrete time-delay difference equations and their invariant sets has received recently a unifying characterization via set factorization [33]. The reader is referred to this work for geometrical details on the Cartesian product of sets in relationship with positive invariance for time-delay systems.

\section{Conclusion}

This paper discusses positive invariance for discrete time-delay systems. Necessary and/or sufficient conditions for the existence of $\mathcal{D}$-invariant sets have been gathered and discussed. The relationship between $\mathcal{D}$ invariance and stability has been studied for discrete delay difference equations (dDDEs). The construction of $\mathcal{D}$-invariant sets via set iterations has been shown to benefit from set convexification, despite the fact that set forward mappings based on the original dDDE lead to a non-convex $\mathcal{D}$-invariant set.

\section{Acknowledgements}

The research leading to these results has benefited from the financial support of the Franco-Italian collaborative research program Galileo 2014.
The first three authors gratefully acknowledge financial support from the European Union's $7^{\text {th }}$ Framework Programme under EC-GA No. 607957 TEMPO - Training in Embedded Predictive Control and Optimization.

\section{References}

[1] F. Blanchini, Set invariance in control, Automatica 35 (1999) 1747-1767.

[2] C. Moussaoui, R. Abbou, J. Loiseau, On bounds of input-output systems. reachability set determination and polyhedral constraints verification, in: 19th IFAC World Congress, Cape Town, South Africa, 2014, pp. 1101211017.

[3] A. Hmamed, A. Benzaouia, M. Ait Rami, F. Tadeo, Positive stabilization of discrete-time systems with unknown delay and bounded controls, in: European Control Conference (ECC), Kos, Greece, 2007, pp. 5616-5622.

[4] D. Mayne, J. Rawlings, C. Rao, P. Scokaert, Constrained model predictive control: Stability and optimality, Automatica 36 (2000) 789-814.

[5] T. L. Santos, A. H. Gonzalez, Stable mpc with reduced representation for linear systems with multiple input delays, in: American Control Conference (ACC), 2015, pp. 238-243.

[6] M. Reble, F. Allgöwer, General design parameters of model predictive control for nonlinear time-delay systems, in: 49th IEEE Conference on Decision and Control (CDC), 2010, pp. 176-181.

[7] S. Olaru, J. A. De Dona, M. M. Seron, F. Stoican, Positive invariant sets for fault tolerant multisensor control schemes, International Journal of Control 83 (12) (2010) 2622-2640.

[8] F. Stoican, S. Olaru, M. M. Seron, J. A. De Dona, Reference governor design for tracking problems with fault detection guarantees, Journal of Process Control 22 (5) (2012) 829-836.

[9] F. Blanchini, S. Miani, Set-theoretic methods in control, Birkhuser, Boston, 2008.

[10] W. Michiels, S.-I. Niculescu, Stability and Stabilization of Time-Delay Systems (Advances in Design \& Control), Society for Industrial and Applied Mathematics, Philadelphia, PA, USA, 2007.

[11] L. Hetel, J. Daafouz, S. Tarbouriech, C. Prieur, Stabilization of linear impulsive systems through a nearly-periodic reset, Nonlinear Analysis: Hybrid Systems 7 (1) (2013) $4-15$.

[12] S. Tarbouriech, Local stabilization of continuous-time delay systems with bounded inputs, in: Stability and control of time-delay systems, Springer, 1998, pp. 302-317.

[13] J.-C. Hennet, A feedback stabilization technique for delay differential systems.

[14] A. Hmamed, A. Benzaouia, H. Bensalah, Regulator problem for linear continuous-time delay systems with nonsymmetrical constrained control., IEEE Transaction on Automatic Control 40 (9) (1995) 1615-1619. doi:10.1109/9.412630.

[15] S. Di Cairano, U. V. Kalabić, I. V. Kolmanovsky, Refer- 
ence governor for network control systems subject to variable time-delay, Automatica 62 (2015) 77-86.

[16] J. C. Keqin Gu, Vladimir L. Kharitonov, Stability of timedelay systems, Springer Science + Business Media, LLC, Birkhäuser, Boston, USA, 2003.

[17] J. E. Normey-Rico, Control of dead-time processes, Springer Science \& Business Media, 2007.

[18] J. L. Avila Alonso, C. Bonnet, H. Özbay, J. Clairambault, S.-I. Niculescu, P. Hirsch, F. Delhommeau, A coupled model for healthy and cancer cells dynamics in acute myeloid leukemia, in: Proceedings of the 19th IFAC World Congress, Vol. 19, 2014, pp. 7529-7534.

[19] S. Miani, A. Morassutti, Switching controllers for networked control systems with packet dropouts and delays in the sensor channel, in: Proc. First IFAC Workshop on Estimation and Control of Networked Systems, 2009, pp. 334-339.

[20] I. Boussaada, H. Mounier, S.-I. Niculescu, A. Cela, Analysis of drilling vibrations: A time-delay system approach, in: 20th IEEE Mediterranean Conference on Control Automation (MED),. IEEE, 2012, pp. 610-614.

[21] A. Seuret, H. Özbay, C. Bonnet, H. Mounier, Lowcomplexity Controllers for Time-delay Systems, Springer, 2014.

[22] G. Samanta, Dynamic behaviour for a nonautonomous heroin epidemic model with time delay, Journal of Applied Mathematics and Computing 35 (1-2) (2011) 161-178.

[23] S. Tarbouriech, J. M. G. d. Silva Jr, Synthesis of controllers for continuous-time delay systems with saturating controls via lmis, IEEE Transactions on Automatic Control, 200045 (1) 105-111.

[24] S. Elaydi, An Introduction to Difference Equations, Springer, 2004.

[25] M. Fisher, B. Goh, Stability results for delayedrecruitment models in population dynamics, Journal of Mathematical Biology 19 (1) (1984) 147-156.

[26] S. Tang, L. Chen, Density-dependent birth rate, birth pulses and their population dynamic consequences, Journal of Mathematical Biology 44 (2) (2002) 185-199.

[27] K. Cooke, A. Ivanov, On the discretization of a delay differential equation, Journal of Difference Equations and Applications 6 (1) (2000) 105-119.

[28] H. Crauel, T. Doan, S. Siegmund, Difference equations with random delay, Journal of Difference Equations and Applications 15 (7) (2009) 627-647.

[29] R. H. Gielen, M. Lazar, I. V. Kolmanovsky, Lyapunov methods for time-invariant delay difference inclusions, SIAM Journal on Control and Optimization 50 (1) (2012) 110-132.

[30] G. Seifert, Positively invariant closed sets for systems of delay differential equations, Journal of Differential Equations 22 (2) (1976) 292-304.

[31] S. Damak, A. Ferhi, V. Andrieu, M. Di Loreto, W. Lombardi, A bridge between Lyapunov-Krasovskii and spectral approaches for stability of difference equations, in: Time Delay Systems, Vol. 11, 2013, pp. 30-35.

[32] R. H. Gielen, M. Lazar, S. V. Raković, Necessary and sufficient Razumikhin-type conditions for stability of delay difference equations, IEEE Transactions on Automatic Control 58 (10) (2013) 2637-2642.

[33] S. Olaru, N. Stanković, G. Bitsoris, S.-I. Niculescu, Low complexity invariant sets for time-delay systems: A set factorization approach, in: Low-Complexity Controllers for Time-Delay Systems, Springer, 2014, pp. 127-139.

[34] M. T. Laraba, S. Olaru, S.-I. Niculescu, G. Bitsoris, Invariant sets for discrete time-delay systems: Set factorization and state representation, in: 19th International Conference on System Theory, Control and Computing (ICSTCC), Cheile Gradistei, Romania, 2015, pp. $7-12$.

[35] N. Athanasopoulos, M. Lazar, On controlled-invariance and stabilization of time-delay systems, in: European Control Conference (ECC), 2014, pp. 778-783.

[36] S. Raković, R. Gielen, Positively invariant families of sets for interconnected and time-delay discrete-time systems, SIAM Journal on Control and Optimization 52 (4) (2014) 2261-2283.

[37] W. Lombardi, S. Olaru, G. Bitsoris, S.-I. Niculescu, Cyclic invariance for discrete time-delay systems, Automatica 48 (10) (2012) 2730-2733.

[38] S. V. Raković, R. H. Gielen, M. Lazar, Construction of invariant families of sets for linear systems with delay, in: Proceedings of the IEEE American Control Conference, 2012, pp. 6246-6251.

[39] M. T. Laraba, S. Olaru, S.-I. Niculescu, F. Blanchini, S. Miani, D. Casagrande, G. Giordano, Set invariance for delay difference equations, in: 12th IFAC Workshop on Time Delay Systems, Ann Arbor (MI), USA, 2015, pp. 215-220.

[40] M. Dambrine, J. P. Richard, P. Borne, Feedback control of time-delay systems with bounded control and state, Mathematical Problems on Engineering 1 (1) (1995) 77-87.

[41] A. Goubet-Bartholomeus, M. Dambrine, J. P. Richard, Bounded domains and constrained control of linear timedelay systems, Journal europeen des systemes automatises 31 (6) (1997) 1001-1014.

[42] J. C. Hennet, S. Tarbouriech, Stability conditions of constrained delay systems via positive invariance, International Journal Of Robust and Nonlinear Control 8 (3) (1998) 265-278.

[43] M. Vassilaki, G. Bitsoris, Constrained feedback control of discrete-time systems described by arma models, In Proceedings of the 1999 European Control Conference, Karlsruhe, Germany.

[44] W. Lombardi, S. Olaru, M. Lazar, S. I. Niculescu, On positive invariance for delay difference equations, In Proceedings of the American Control Conference (2011) 36743679 .

[45] W. Lombardi, A. Luca, S. Olaru, S.-I. Niculescu, On the polyhedral set invariance conditions for time-delay systems, in: Proceedings of the 18th IFAC World Congress, Milano, Italy, 2011, pp. 308-313.

[46] N. Stanković, S. Olaru, S. I. Niculescu, Further remarks on asymptotic stability and set invariance for linear delay difference equations, Automatica 50 (8) (2014) 2191-2195.

[47] A. Y. Aleksandrov, O. Mason, Diagonal LyapunovKrasovskii functionals for discrete-time positive systems with delay, Systems \& Control Letters 63 (2014) 63-67.

[48] G. Bitsoris, N. Athanasopoulos, L. Dritsas, Stability, positive invariance and design of constrained regulators for networked control systems, International Journal of Control 85 (10) (2012) 1401-1413.

[49] W. Heemels, N. Van de Wouw, R. Gielen, M. Donkers, L. Hetel, S. Olaru, M. Lazar, J. Daafouz, S. Niculescu, Comparison of overapproximation methods for stability 
analysis of networked control systems, in: Proceedings of the 13th ACM International Conference on Hybrid systems: computation and control, 2010, pp. 181-190.

[50] A. Halanay, V. Rasvan, Stability and stable oscillations in discrete time systems, Vol. 2, CRC Press, 2000.

[51] K. Aström, B. Wittenmark, Computer-controlled systems: theory and design., Prentice Hall, 1997.

[52] W. Lombardi, Constrained control for time-delay systems., Ph.D. thesis, Supélec (2011).

[53] R. H. Gielen, M. Lazar, S. Olaru, Set-induced stability results for delay difference equations, In Time Delay Systems: Methods, Applications, and New Trends, Springer series Lecture Notes in Control and Information Sciences (423) (2012) 73-84.

[54] J.-C. Hennet, S. Tarbouriech, Stability and stabilization of delay differential systems., Automatica 33 (3) (1997) 347-354. doi:10.1016/S0005-1098(96)00185-9.

[55] G. M. Ziegler, Lectures on polytopes, Vol. 152, Springer Science \& Business Media, 1995.

[56] R. Schneider, Convex bodies: the Brunn-Minkowski theory, no. 151, Cambridge University Press, 2013.

Mohammed-T. Laraba was born in Mila, Algeria on August 4, 1990. He received his M.Sc. degree in Control and Signals Processing from Paris-Sud University, France in 2014. Thereafter, he started working towards a Ph.D. degree in the Laboratory of Signals and Systems at CentraleSupélec. He is working as an Early Stage Researcher (ESR) in Marie Curie Initial Training Network on Embedded Model Predictive Control and Optimization (ITN-TEMPO). His research interests include analysis and control synthesis for dynamical systems under constraints, setvalued systems analysis and synthesis, time-delay systems, model predictive control.

Sorin Olaru is a Professor in CentraleSupélec, member of the CNRS Laboratory of Signals and Systems and of the INRIA team DISCO, all these institutions being part of the Paris-Saclay University in France. His research interests are encompassing the optimization-based control design, set-theoretic characterization of constrained dynamical systems as well as the numerical methods in control. He is currently involved in research projects related to embedded predictive control, fault tolerant control and time-delay systems.

Silviu-Iulian NICULESCU received the B.Sc. degree from the Polytechnical Institute of Bucharest, Romania, the M.Sc., Ph.D. degrees, in automatic control, from INP Grenoble, France and the French habilitation from Universit Technologique de Compigne, France. From September 2006, he joined L2S (Laboratory of Signals and Systems), Gif-sur-Yvette, where he is currently a CNRS research director (senior researcher) and the head of the laboratory. Dr.
Niculescu is the author or co-author of 9 books and more than 400 book chapters or scientific papers. Since 2012, he is the founding editor and the scientific responsible of a new Springer (book) series: Advances in Delays in Dynamics (ADD@S). Dr. Niculescu was awarded the CNRS Silver and Bronze Medals for scientific research, and the Ph.D. Thesis Award from INP Grenoble (France) in 2011, 2001, and 1996, respectively. His research interests include delay systems, robust control, operator theory, and numerical methods in optimization, and their applications to the design of engineering systems.

Franco Blanchini was born on 29 December 1959, in Legnano (Italy). He is the Director of the Laboratory of System Dynamics at the University of Udine. He was Program Vice-Chairman of the Conference Joint CDC-ECC 2005, Seville, Spain; Program ViceChairman of the Conference CDC 2008, Cancun, Mexico; Program Chairman of the Conference ROCOND, Aalborg, Denmark, June 2012 and Program Vice-Chairman of the Conference CDC 2013, Florence, Italy. He is co-author of the book "Set theoretic methods in control", Birkhäuser. He is the recipient of the 2001 ASME Oil \& Gas Application Committee Best Paper Award and of the 2002 IFAC survey paper prize for the article "Set Invariance in Control - a survey", Automatica, November 1999. He has been an Associate Editor for Automatica from 1996 to 2006. Since 2011, he has been an Associate Editor for IEEE Transactions on Automatic Control. Giulia Giordano received her B.Sc. and M.Sc. degrees summa cum laude in Electrical Engineering, in 2010 and 2012 respectively, from the University of Udine, Italy, where she is currently working towards a Ph.D. with a focus on Systems and Control Theory. She visited the Control and Dynamical Systems group at the California Institute of Technology, Pasadena (CA), USA, in 2012 and the Institute of Systems Theory and Automatic Control at the University of Stuttgart, Germany, in 2015. Her main research interests include analysis of biological systems and control of network systems.

Stefano Miani was born in Parma, Italy, in 1967. He received the electrical engineering Laurea degree and the Ph.D. degree in control engineering from the University of Padova, Italy, in 1993 and 1996, respectively. In 1997 he worked at the University of Padova as an Assistant Professor. Since November 1998 he has worked at the University of Udine, Italy, where he currently holds an Associate Professor position. He 
has been member of editorial boards and organizing committees of international journals and conferences and he is currently member of the "IFAC Technical Committee on Robust Control" and Associate Editor for the IEEE Transactions on Automatic Control. His research results, developed in the areas of constrained control, $l_{\infty}$ disturbance attenuation problems, gain scheduling control, uncertain production-distribution systems via set-valued techniques, and switching controllers, have been published in more than 80 international papers and an international book.

Daniele Casagrande was born in Belluno, Italy, in 1973. He received the "Laurea" degree in electronic engineering in 1999 and the $\mathrm{PhD}$ in information engineering in 2006, both from the University of Trieste, Italy. In 2006 he was research associate at the Institute of Cybernetics of the Tallinn University of Technology, Estonia. Between 2006 and 2007 he was research associate at the Department of Electrical, Electronic and Computer Engineering of the University of Trieste. In 2008 he was research associate at the Department of Electrical and Electronic Engineering of Imperial College London, UK. Since 2008 he is with the Department of Electrical, Managing and Mechanical Engineering of the University of Udine, Italy, where he is currently an assistant professor in optimization. His current research interests are focused on stability of switched system, Hamiltonian systems, LPV stability, nonlinear control and optimization. 